\title{
For bone metastatic patients, further sailing, better service
}

Author/Photo: Yi Cheng, from Tongji Cancer Center, Tongji Hospital, Tongji Medical College, Huazhong University of Science and Technology, Wuhan 430030, China

Received: 18 June 2010 / Revised: 20 June 2010 / Accepted: 23 June 2010

(c) Huazhong University of Science and Technology and Springer-Verlag Berlin Heidelberg 2010

On July 9th, 2010, the second scene of speech and advisory service for SAIL in 2010 was delivered in the classroom of Tongji Cancer Center. Compared to this public benefic activity in 2009, more patients and their relatives learned the fundamental and latest information about the bone metastasis.

The scene of speech was given by Professor Qian Chu, from Tongji Cancer Center. She introduced the cause and mechanism of bone metastasis, the risk, the clinical manifestation, the diagnosis, and what was the most detailed, the treatment. She pointed that, since more patients could survive for a longer time with the help of the latest anticancer therapy, the oncological doctor should pay more attention to the complications of tumor, such as bone metastasia, which could induce pathological bone fracture, spinal compression and malignant hypercalcemia. The comprehensive treatment should be considered, including analgesic drugs, bisphosphonates, chemotherapy, radiotherapy, surgery and rehabilitation therapy.

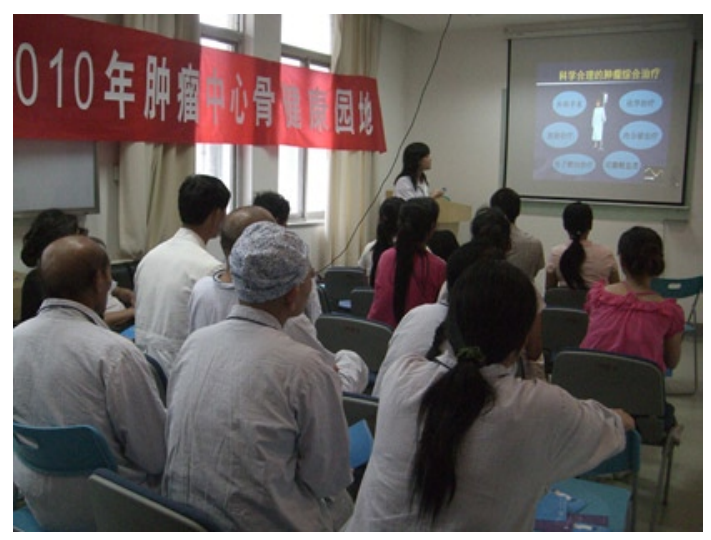

Fig. 2 The audiences were listening carefully

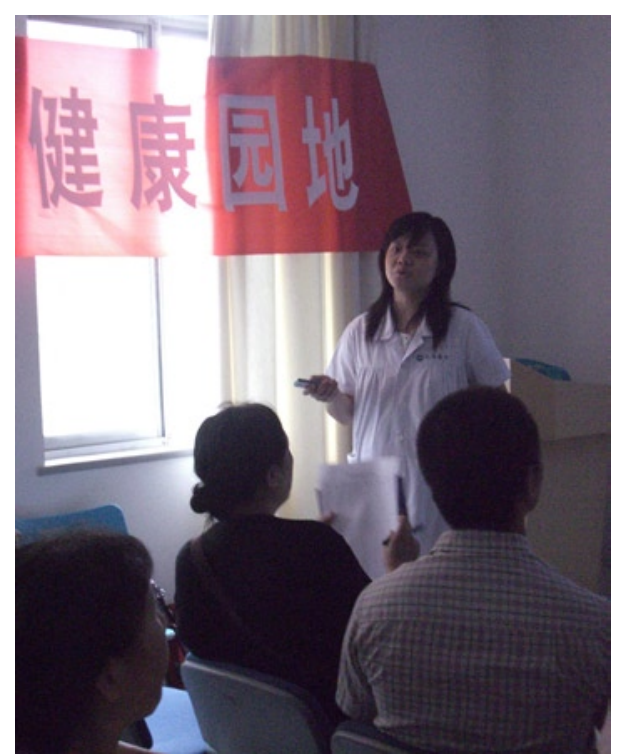

Fig. 1 Professor Qian Chu was delivering a speech

After the speech, an advisory service was held in the classroom. What was more, every audience got a booklet about the bone metastasis, which was provided by Novartis.

SAIL, which means "Survival, Anti-tumor, Independent Life", was launched in 2008 by CRPC. This public benefic activity aims the patients suffering bone metastasis. With the help of speeches, advisory services and questionnaire investigation, it heightens the patients' and doctors' proper understanding to the disease. Now it has been three years, and more and more patients benefit from the activity. CRPC would keep "sailing" the activity further, for bone metastatic patients. 